\title{
The discovery of the weak neutral currents
}

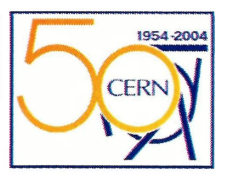

Thirty years have passed since the discovery of weak neutral currents in the Gargamelle bubble chamber at CERN. Today the huge impact of this discovery on CERN, the field of high-energy physics and beyond, is highly visible; then, however, it was received with great scepticism both by CERN and the physics community.

Shortly after the Siena Conference in 1963, André Lagarrigue, André Rousset and Paul Musset worked out a proposal for a neutrino experiment that aimed to increase the event rate by an order of magnitude. This meant building a large heavy-liquid bubble chamber, later named Gargamelle (figure 1), and also forming a large collaboration. The core of the team consisted of members of Orsay, the École Polytechnique and the neutrino experiments with the CERN NPA (Nuclear Physics Apparatus) division $1 \mathrm{~m}$ bubble chamber, which were just finishing. In the end, the collaboration consisted of seven European laboratories and also included guests from Japan, Russia and the US.

\section{The challenge}

At the end of the 1950s V-A theory was the "standard model" of weak interactions. Its major drawback was its bad high-energy behaviour, which prompted various ideas to cure the problem of infinities. Guided by quantum electrodynamics, a gauge theory, attempts were made to construct a gauge theory of weak interactions, and in the mid-1960s the hypothesized charged intermediate vector boson $\left(\mathrm{W}^{ \pm}\right)$was complemented with a neutral partner to achieve the required cancellations. The invention of the Higgs mechanism solved the problem of having both a gauge theory and massive mediators of weak interactions. The progress made by Sheldon Glashow, Abdus Salam and Steven Weinberg was completed by the work of Martinus Veltman and Gerard 't Hooft, which proved the renormalizability of the theory. So, as 1971 turned to 1972 , a viable theory of weak interactions that claimed weak neutral currents as a crucial ingredient was proposed, challenging the experimental groups to provide "yes" or "no" as an answer to the question "do neutral currents exist?".

By that time two neutrino experiments were running, Gargamelle at the CERN Proton Synchrotron and the HPWF (Harvard, Pennsylvania, Wisconsin, Fermilab) counter experiment at what is now Fermilab. Both were confronted with this challenge without preparation. The searches for neutral currents in previous neutrino experiments

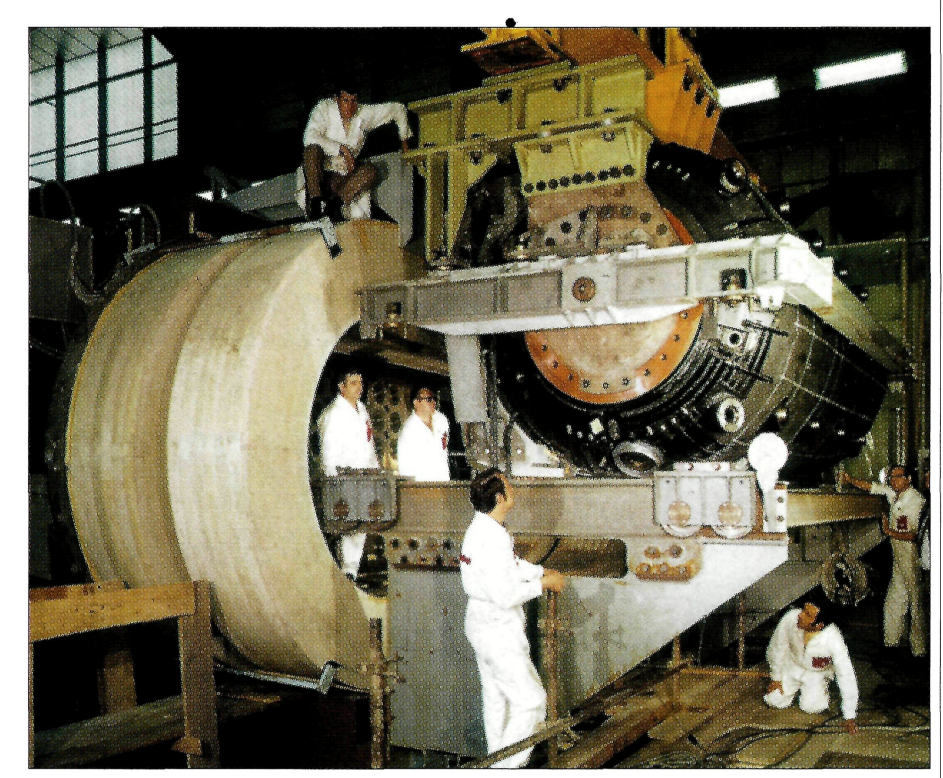

Fig. 1. The Gargamelle heavy-liquid bubble chamber, installed into the magnet coils, at CERN in 1970.

\section{Table 1 \\ No. of neutral-current candidates v-exposure $\bar{v}$-exposure 102 64 No. of charged-current candidates 428 148}

resulted in discouragingly low limits, and it was somehow commonly concluded that no weak neutral currents existed. In fact, during the two-day meeting in November 1968 in Milan, where the Gargamelle collaboration discussed its future neutrino programme, the words "neutral current" were not even mentioned. On the contrary, the real highlight that attracted the interest of all was the recent observation of the proton substructure at SLAC, provoking the question of what structure would be revealed by the $\mathrm{W}$ in the neutrino experiments as opposed to the photon in electron-proton scattering.

Although the quest for neutral currents had been ignored, Gargamelle could meet the challenge once the matter of their discovery became urgent at the beginning of 1972 . That is to say, scanning and event classification followed the same rules as established in $\triangleright$ 


\section{0 ${ }^{\mathrm{TH}}$ ANNIVERSARY}

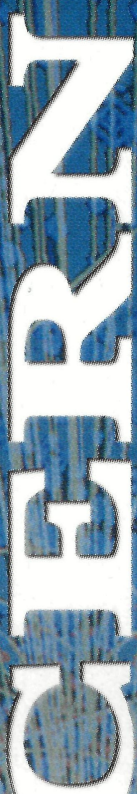

We wish you another 50 years and much success for the

LHC program! $\circ \otimes$

Thank you for choosing CES and our real-time data acquisition equipment.

$W_{\text {e have been serving } C E R N \text { for }}$ nearly 25 years and have kept the physics breed alive at CES throughout the generations.

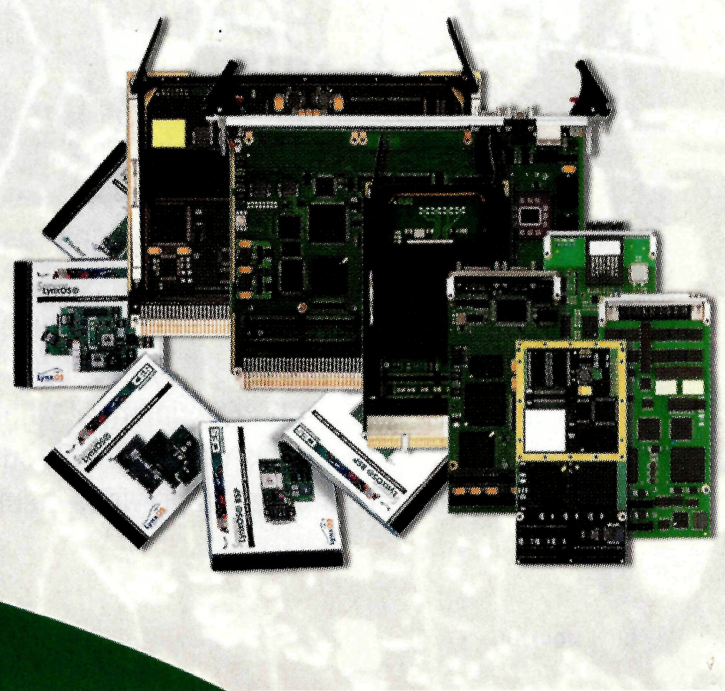


the previous, NPA bubble-chamber experiment. There was no muon identification, since weak processes were supposed always to transform an initial-state neutrino into a final-state muon. Consequently, there was an unavoidable background of events in which a charged hadron leaves the visible volume of the chamber without visible interaction, thus faking a muon. Events with a muon candidate were collected in one category, $A$, while events consisting of secondaries that were all identified as hadrons were collected in a second category, B. These category-B events, the so-called neutron stars $\left(n^{*}\right)$, were thought to arise when undetected upstream neutrino interactions emitted a neutron that interacted in the chamber. It was then easy to deduce from these events the fraction that did not interact, thus simulating a muon, and to subtract them from the observed number of events in category $A$.

So, if weak neutral currents indeed existed, they would have induced events consisting of hadrons only, just as the $n * s$, and they would be waiting to be discovered as part of category B. Consequently, their investigation could be undertaken without any loss of time. The main task was then to find ways of distinguishing neutrino-induced from neutron-induced events.

\section{Three hot months in 1973}

The measurements of the inclusive neutral-current (NC) candidates were carried out between September 1972 and March 1973. The observation of an isolated electron in the anti-neutrino film, interpreted as an elastic weak neutral-current interaction on an electron, generated great excitement and inspired the efforts to check carefully each neutral-current candidate (Hasert et al. 1973a). For comparison, a charged-current (CC) sample was collected, where the same criteria were applied to the hadrons as for the neutral-current candidates. In particular, the total deposited hadron energy had to exceed $1 \mathrm{GeV}$. This severe cut was intended to keep the number of $n *$ small.

At the collaboration meeting in March 1973 at CERN it looked as though a discovery was at hand. The number of neutral-current candidates was encouragingly large, as seen in table 1 on p21 (Hasert et al. 1973b). Their spatial distributions, as shown in figure 2 , suggested first that the vertex distribution of the neutral-current candidates is neutrino-like, since it is flat like the charged-current events; and second that there is no indication of an exponentially falling distribution at the beginning of the chamber, as should be expected if the neutral-current candidates were dominantly induced by neutrons. Both arguments were corroborated by a Monte Carlo simulation of the Orsay group based on the simplifying assumption that upstream neutrino-induced neutrons enter the chamber directly along the neutrino direction.

Yet Jack Fry and Dieter Haidt contested that both arguments were not cogent for two strong reasons. First, the neutrino flux has a broad radial extension, which causes neutrino interactions in the coils surrounding the chamber and thus a flux of neutrons that enters the fiducial volume uniformly from the side. Second, high-energy neutrons generate a cascade, implying that the longer energydependent cascade length, rather than the interaction length, defines the relevant measure for the number of background events. Thus, it was unclear whether the neutral-current candidates really contained a novel type of neutrino-induced event or whether they

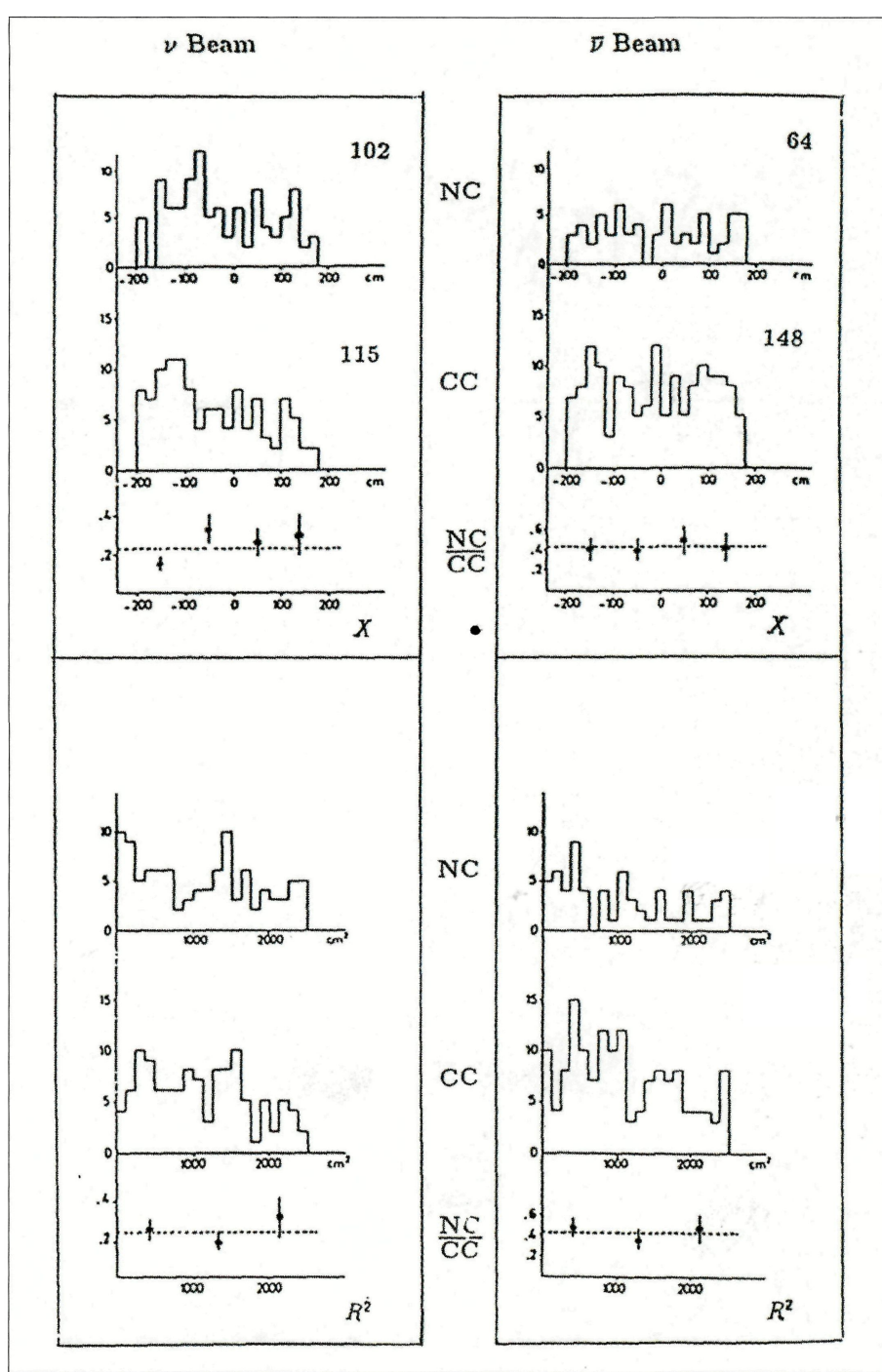

Fig. 2. The spatial distributions of neutral (NC) and chargedcurrent (CC) candidates, as measured in 1973. $X$ is the vertex position along the chamber axis and $R$ is the radial position.

were merely the expected, good-old neutron-induced stars.

In this situation a detailed neutron background calculation was indispensable. The programme had to take into account the geometry and matter distribution of the chamber, the magnet coils and the shielding, the neutrino flux in energy and radial distributions, the dynamics of the final state, and most of all the neutral hadron cascade. The demanding task consisted of describing realistically the complex final hadron state. The breakthrough was achieved when it became clear that only fast final-state nucleons can generate a cascade and eventually lead to an induced neutron background event satisfying the energy requirement and that, furthermore, the cascade is linear. All the ingredients to the programme were backed up by data, so the predictions did not depend upon free parameters. This ambitious programme (Fry and Haidt 1975) was set up, carried through in the following months, and led in July 1973 to the undisputable conclusion that the neutron-induced background explained only a small fraction of the neutral-current candidates; thus a new effect could be claimed and published (Hasert et al. 1973b). In an independent check, Antonino Pullia exploited the $\triangleright$ 


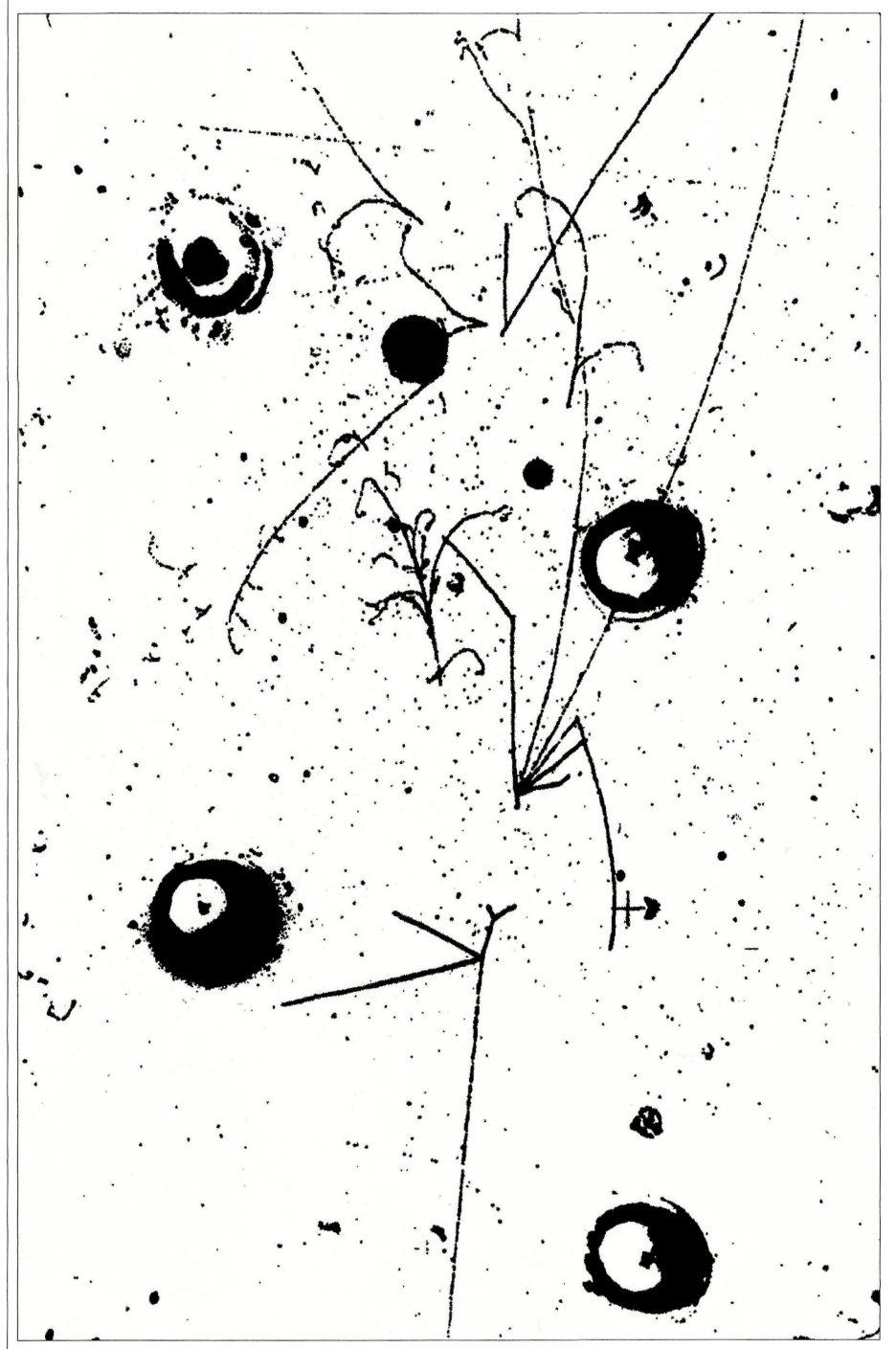

Fig. 3. A $7 \mathrm{GeV}$ proton enters the Gargamelle bubble chamber from below and induces a three-step neutron cascade.

spatial distributions of neutral current and charged-current candidates, providing further evidence that the neutral-current sample was not dominated by neutron stars (Hasert et al. 1974).

\section{Attack and victory}

The new results were presented at the Electron-Photon Conference one month later at Bonn, together with the results of the HPWF experiment. At the end of the conference, Chen-Ning Yang announced the existence of weak neutral currents as the highlight of the meeting.

Shortly afterwards, the HPWF collaboration modified their apparatus with the net result that the previously observed signal of neutral currents disappeared. This news quickly reached CERN, where it had a dismaying effect and was a cause for distrust of the Gargamelle result. The opponents focused their criticism on the neutron background calculation and in particular on the treatment of the neutron cascade. Although the members of the Gargamelle collaboration withstood all the critical questions, the willingness to accept the validity of the Gargamelle observation had to wait until the end of the year. In a special exposure of Gargamelle to shots of protons with fixed momentum, the prediction of the cascade pro- gramme was verified quantitatively and unambiguously by the direct observation of proton-induced cascades in the chamber (figure 3). The results were presented at the American Physical Society conference in Washington in April 1974 (Haidt 1974).

One year after the discovery, at the time of the conference in London in June 1974, overwhelming confirmation for the existence of weak neutral currents came from Gargamelle itself with twice the original statistics (Hasert et al. 1974). In the meantime the HPWF collaboration had elucidated the reason why they lost the signal and also affirmed weak neutral currents. Further confirmation came from the new counter experiment of the California Institute of Technology and Fermilab (CITF) collaboration and from the observation of neutral-current-induced single pion events in the $12 \mathrm{ft}$ bubble chamber at Argonne.

\section{The impact}

The discovery of weak neutral currents crowned the long-range neutrino programme initiated by CERN at the beginning of the 1960 s and brought CERN a leading role in the field. The new effect marked the experimental beginning of the Standard Model of electroweak interactions and triggered huge activity at CERN and all over the world, both on the experimental and theoretical sides. The most immediate success was the prediction of the mass value of the elusive intermediate vector boson, W, on the basis of the GlashowSalam-Weinberg model, combined with the first measurements of the weak mixing angle $\theta_{\mathrm{W}}$. This led to the idea of building a protonantiproton collider, which was later realized at CERN and brought about the observation at CERN of the mediators of the weak force, the W and Z (CERN Courier April 2004 p13). The neutrino experiments at the CERN Super Proton Synchrotron increased their precision to the point that the first test of weak radiative corrections was enabled. The continuously increasing amount of knowledge on weak interactions justified building the Large Electron Positron collider, LEP, which with its high intensity reached sufficient precision at the energy range of the $Z$ mass and beyond to test electroweak theory at the quantum level (CERN Courier May 2004 p21). All the results combined make the search for the Higgs, the last element of the electroweak Standard Model, a central issue for the Large Hadron Collider.

- This article is based on a talk at the symposium held at CERN in September 2003, "1973: neutral currents, 1983: W and Z bosons. The anniversary of CERN's discoveries and a look into the future." The full proceedings have been published as volume 34 issue 1 of The European Physical Journal C, and as a book, Prestigious Discoveries at CERN, by Roger Cashmore, Luciano Maiani and Jean-Pierre Revol (Springer ISBN 3540207503, September 2004).

\section{Further reading \\ W F Fry and D Haidt 1975 CERN Yellow Report 75-01. \\ D Haidt 1974 Contribution to the American Physical Society \\ Conference at Washington, April 1974. \\ F J Hasert et al. 1973a Phys. Lett. 46B 121. \\ F J Hasert et al. 1973b Phys. Lett. 46B 138. \\ F J Hasert et al. 1974 Nucl. Phys. B73 1.}

\section{Dieter Haidt, DESY.}



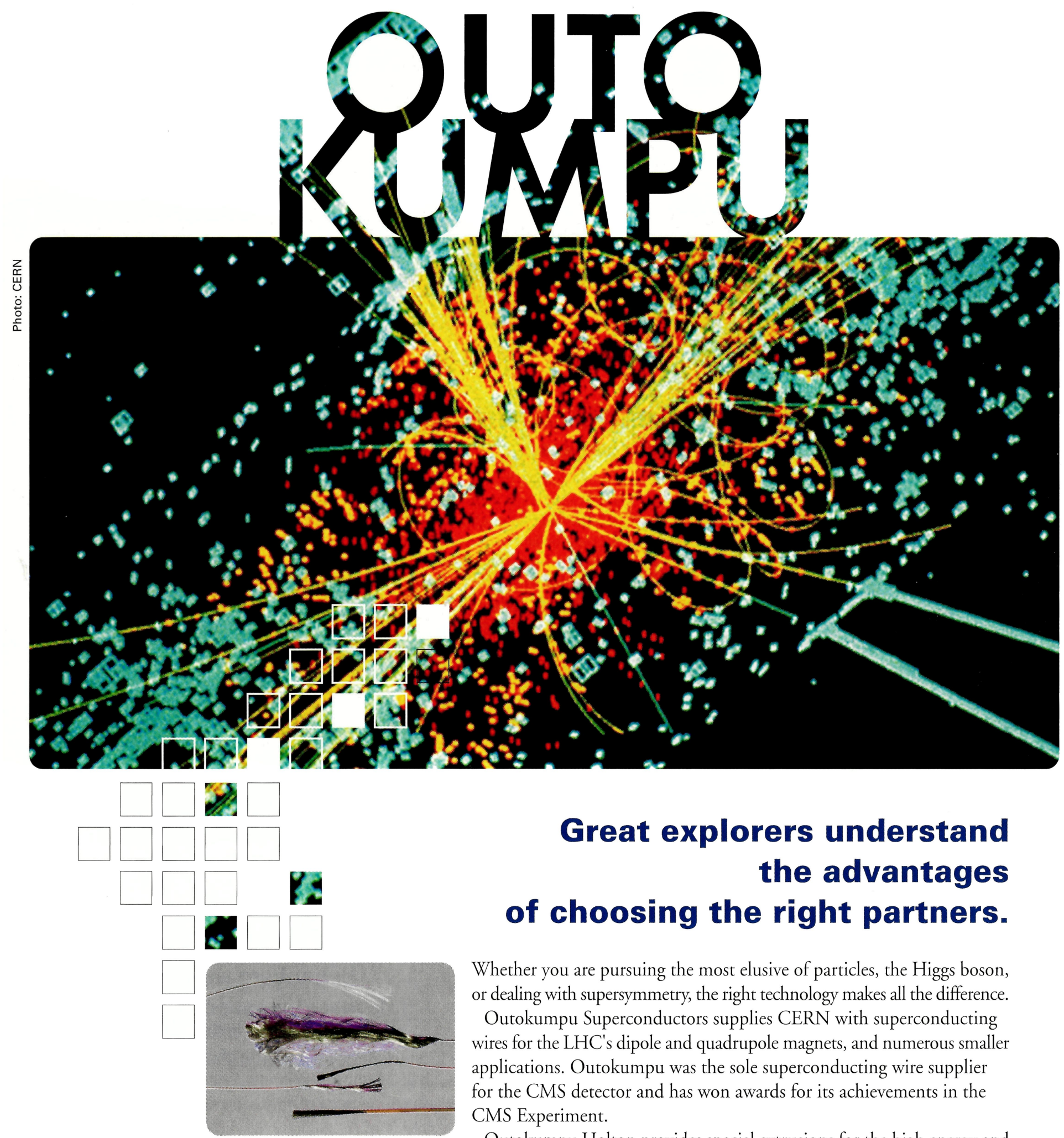

Whether you are pursuing the most elusive of particles, the Higgs boson, or dealing with supersymmetry, the right technology makes all the difference. Outokumpu Superconductors supplies CERN with superconducting wires for the LHC's dipole and quadrupole magnets, and numerous smaller applications. Outokumpu was the sole superconducting wire supplier for the CMS detector and has won awards for its achievements in the CMS Experiment.

Outokumpu Holton provides special extrusions for the high energy and particle physics applications, including large lengths of aluminium conductor for the LHCb, ALICE experiments, and for the replacement of the Proton Synchrotron (CPS) main magnet units. Also aluminium stabilised superconductor for the AMS 02 detector for the International Space Station.

The Outokumpu factor means giving you the best in performance enhancing metals and technology. It is an honour for us to be part of this great scientific adventure. 


\section{New Books from

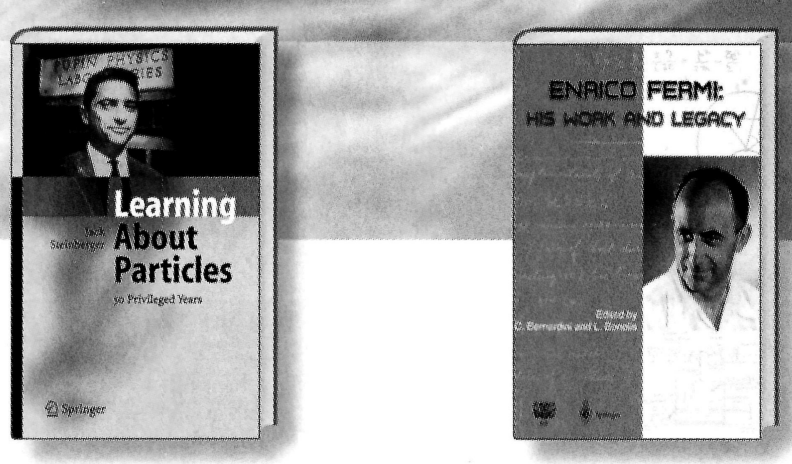 \\ Springer

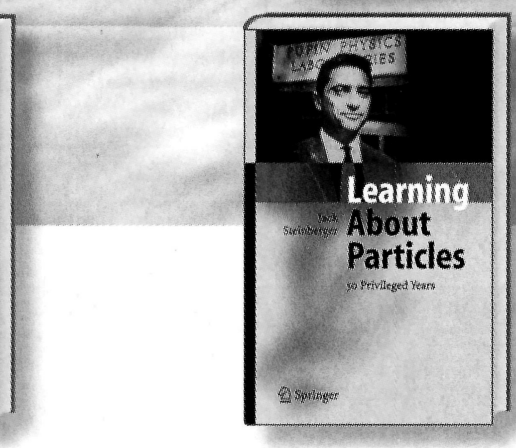

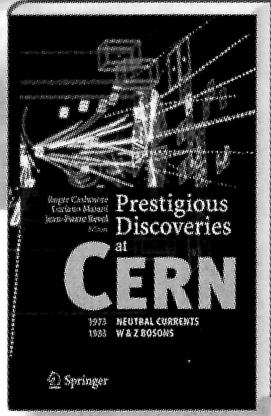

J. Steinberger

L. Maiani; J.-P. Revol (Eds.)

\section{Prestigious Discoveries at CERN}

1973 Neutral Currents

1983 W \& Z Bosons

The discoveries of neutral currents and of the $\mathrm{W}$ and $\mathrm{Z}$ bosons marked a watershed in the history of CERN They established the validity of the electroweak theory and convinced physicists of the importance of renormalizable non-Abelian gauge theories of fundamental interactions. The articles collected in this book have been written by distinguished physicists who contributed in a crucial way to these developments. The book provides a historical account of those discoveries and of the construction and testing of the Standard Model. It also contains a discussion of the future of particle physics and gives an updated status of the LHC and its detectors currently being built at CERN.

2004. Approx. 200 p. 114 illus., 88 in color. Hardcover

$€$ 39.95; sFr 73; £ 30.50 ISBN 3-540-20750-3

\section{Castell; O. Ischebeck (Eds.)}

\section{Time, Quantum and Information}

This collection of essays presented to Carl Friedrich von Weizsäcker on the occasion of his 90th birthday addresses a wide readership interested in astronomy, physics, and the history and philosophy of science. The articles treat subjects such as the social responsibility of scientists, thermonuclear processes in stars and stellar neutrinos, turbulence and the emergence of planetary systems. Furthermore, considerable attention is paid to the unity of nature, the nature of time, and to information about, and interpretation of, the structure of quantum theory, all important philosophical problems of our times. The last section describes von Weizsäcker's ur-hypothesis and how it will theoretically permit the construction of particles and interactions from quantized bits of information.

1st ed. 2003. Corr. 2nd printing 2004. XIV, 456 p. Hardcover $€$ 49.95; sFr 88.50; £ 38.50 ISBN 3-540-44033-X

\section{Learning about particles - 50 privileged years}

Embedded in an autobiographic framework, this book retraces vividly and in some depth the golden years of particle physics as witnessed by one of the scientists who made seminal contributions to the understanding of what is now known as the Standard Model of particle physics well beyond a survey of interest to historians of sciences and researchers in the field. This book is a must for all students and young researchers who have learned about the theoretical and experimental facts that make up the standard model through modern textbooks only. It will provide the interested reader with a first hand account and deeper understanding of the multilayered and sinuous development that finally led to the present architecture of this theory.

2004. Approx. 250 p. Hardcover $€ 39.95 ; s F r 73 ; £ 30.50$ ISBN 3-540-21329-5

\section{J. Magill; J. Galy}

\section{Radioactivity - Radionuclides - Radiation}

\section{Featuring the Universal Nuclide Chart}

Radioactivity - Radionuclides - Radiation provides professionals and students with a comprehensive background in the three Rs of nuclear science. It is suitable for students in the related fields of nuclear and radiochemistry, nuclear medicine, health physics, environmental sciences, nuclear and astrophysics. The book covers recent developments in the areas of exotic decay modes (bound beta decay of 'bare' or fully ionized nuclei), laser transmutation, nuclear forensics, radiation hormesis and the LNT hypothesis, and alpha-immunotherapy. Atomic mass data for ove 3000 nuclides from the most recent (2003) evaluation are included. The book includes a CD-ROM containing the Universal Nuclide Chart - an interactive, platform independent JAVA-program for displaying basic nuclear data, decay processes and neutron reactions.

2004. Approx. 195 p. 162 illus. With CD-ROM. Softcover $€$ 49.95; sFr 88.50; $£ 38.50$ ISBN 3-540-21116-0
C. Bernardini; L. Bonolis (Eds.)

\section{Enrico Fermi: His Work and Legacy}

Enrico Fermi's scientific work, noted for its originality and breadth, has had lasting consequences throughout modern science. Written by close colleagues as well as scientists whose fields were profoundly influenced by Fermi, the papers collected here constitute a tribute to him and his scientific legacy. They were commissioned on the occasion of his 100th birthday by the Italian Physical Society and confirm that Fermi was a rare combination of theorist, experimentalist, teacher and inspiring colleague. The book is organized into three parts: three biographical overviews by close colleagues, replete with personal insights; fourteen analyses of Fermi's impact by specialists in their fields, spanning physics, chemistry, mathematics, and engineering; and a year-by-year chronology of Fermi's scientific endeavors.

2004. XIII, 380 p. Hardcover $€$ 39.95; sFr 73; $£ 30.50$ ISBN 3-540-22141-7

\section{G. W. Buschhorn; J. Wess (Eds.)}

\section{Fundamental Physics - Heisenberg and Beyond}

Werner Heisenberg Centennial Symposium "Developments in Modern Physics"

Quantum mechanics, formulated by Werner Heisenberg in 1925, belongs among the greatest achievements of physics. "Fundamental Physics: Heisenberg and Beyond" combines personal tributes to Werner Heisenberg with assessments of his impact on current and future developments in physics. The first part presents two essays commemorating Werner Heisenberg's 100th birthday, and these are complemented by a short and nicely illustrated biographical note in the appendix. In the second part, incisive articles by ten world-leading scientists explain important develop ments in fundamental physics to a broader community of interested scientists.

2004. X, 190 p. 44 Illus. Hardcover $€$ 49.95; sFr 88.50; £ 38.50 ISBN 3-540-20201-3

\section{Super savings at Physics Sale springeronline.com/physicssale}

